\title{
Trabajo colaborativo y la ecología del aprendizaje
}

Rosa A. Barrera ${ }^{1}$, Rosa M. Montaño', Pedro E. Marín ${ }^{1}$ y Jorge E. Chávez ${ }^{2}$

(1) Departamento de Matemática y Ciencia de la Computación, Universidad de Santiago de Chile, Las Sophoras 175, Estación Central, Chile. (correo-e: rosa.barrera@usach.cl; rosa.montano@usach.cl; pedro.marin@usach.cl) (2) Facultad de Educación, Universidad Andrés Bello. Santiago, Edificio Bellavista 121, Providencia, Chile (correo-e:; jorge.chavez@unab.cl)

Recibido Mar. 12, 2021; Aceptado May. 10, 2021; Versión final Jul. 8, 2021, Publicado Dic. 2021

\section{Resumen}

El propósito de este estudio es determinar perfiles de comportamiento, considerando elementos de la ecología del aprendizaje, en estudiantes de primer año de enseñanza superior que participan en foros de discusión en ambientes colaborativos digitales. Para establecer dichos perfiles, se analizan las contribuciones de los estudiantes según dos dimensiones: 1) la clasificación del mensaje en cuanto a tipo de contenido y profundidad y 2) las variables de contexto como día, hora y tema de la contribución. Se aplica un análisis descriptivo-cuantitativo. La muestra está conformada por 43 estudiantes de un curso de computación. Los principales resultados apuntan a identificar los horarios y días que inciden en la profundidad del aprendizaje. Se observa que las contribuciones con mayor nivel de profundidad son después de las 19:00 horas. Como conclusión, está investigación identifica ciertos perfiles de comportamiento de la ecología del aprendizaje que favorecen los resultados de un aprendizaje profundo en estudiantes.

Palabras clave: foros colaborativos; enseñanza superior; nueva ecología; computador; aprendizaje profundo

\section{Collaborative work and learning ecology}

\begin{abstract}
The purpose of this study is to develop behavioral profiles, by considering elements of learning ecology, of first-year higher education students that participate in collaborative discussion forums on a digital environment. To determine student profiles, student inputs are analyzed according to two dimensions: 1) message type (content and depth) and 2) context variables that include day of the week, time of the day, and input topic. A descriptive-quantitative analysis is applied. The sample is composed by 43 students enrolled in a computer science course. The results aim to identifying the times of the day and the days of the week that most affect deep learning. Student inputs with the greatest level of depth were attained after 7:00 p.m. In conclusion, this research study identifies learning ecology behavioral profiles that improve deep learning in students.
\end{abstract}

Keywords: collaborative forums; higher education; new ecology; learning; computer; deep learning 


\section{INTRODUCCIÓN}

Los procesos de enseñanza aprendizaje contemporáneos en educación superior, deben estar insertos en una estructura digital que apoye la inclusión en un mundo cada vez más globalizado, donde la ecología del aprendizaje complementa una visión más global del contexto en el cual se produce el aprendizaje, dando una mirada dentro y fuera del aula en la construcción del conocimiento (González-Sanmamed et al, 2020; Dado y Bodemer, 2017; Coll, 2013), en este marco, los entornos virtuales colaborativos de aprendizaje (CSCL) son primordiales, ya que por su naturaleza pueden integrar en forma natural la ecología del aprendizaje, donde prima un aprendizaje personalizado. En este paradigma, dónde los CSCL tienen un enorme potencial para facilitar procesos de aprendizaje profundo (Radkowitsch, 2020; VanOostveen et al, 2016), aun es necesario indagar en interacciones que se produzcan en estos entornos, que ayuden a comprender lo que afecta al aprendizaje colaborativo (Hernández et al, 2019; Arras-Vota et al; 2021). La ecología del aprendizaje proporciona estas herramientas, al tener un enfoque centrado a lo largo y ancho de la vida, proporcionando un marco para saber en que contexto y que elementos emplean los estudiantes para aprender.

En cuanto a los resultados de investigaciones, el foro sigue siendo una de las herramientas más utilizadas en CSCL, por sus características de interactividad, interacción y negociación, lo que hace al foro una herramienta adecuada a este tipo de proceso (Chávez et al, 2016). En general, la calidad de las contribuciones en la mayoría de los casos no es suficiente para lograr un aprendizaje profundo por parte de los participantes (Duque et al, 2018; Stahl, 2015) lo que hace al foro una herramienta de estudio, no solo del aprendizaje profundo, sino para analizar algunos aspectos centrales de la ecología del aprendizaje, como lo son: los contextos que utiliza el individuo, como por ejemplo lugar y tiempo de estudio, entre las más relevantes.

Por otro lado, al incorporar modelos CSCL en educación superior, en forma innata son acompañados por enfoques sociales de aprendizaje (Dado y Bodemer, 2017), dónde la ecología del aprendizaje está siendo investigada hace unas décadas, entregando elementos complementarios al trabajo colaborativo que enriquecen la visión de una educación moderna en educación superior (González-Sanmamed et al; 2020; Hernández et al, 2019). De las investigaciones, se identifican tres características de la ecología del aprendizaje, la primera de ellas hace referencia a que el aprendizaje se produce "a lo ancho de la vida" y no sólo a lo largo. La segunda, vinculada igualmente a la penetración y la presencia creciente de la tecnología digital en prácticamente todos los contextos de actividad de las personas, tiene que ver con la falta de una separación nítida entre los diferentes espacios físicos e institucionales en los cuales puede tener lugar el aprendizaje. Por último, la tercera característica apunta a la puesta en valor de las trayectorias individuales de aprendizaje como vía de acceso al conocimiento en la sociedad de la información.

Sin duda, estos modelos son complejos de implementar y para aportar en este campo, la investigación de este artículo contribuye desde el punto de vista de la herramienta del foro como potencial facilitador del aprendizaje colaborativo y como además puede favorecer algunos de los aspectos de la ecología del aprendizaje. Más específicamente, el problema de investigación que afronta este trabajo es que los estudios no han mostrado con claridad que factores son los que influyen en la característica del mensaje que los estudiantes realizan en sus contribuciones en los foros, principalmente, no se ha analizado las similitudes y diferencias de las condiciones del entorno -tiempo, espacio y lugar- que experimentan los estudiantes.

Esto es relevante, ya que estos constructos se han estudiado por separado, pero no hay trabajos en determinar perfiles de acuerdo a como y cuando se produce el aprendizaje, de ahí, las preguntas emergentes son ¿Cuáles son las características de los mensajes que surgen en contextos de foros colaborativos, considerando tipo y profundidad del aporte? ¿Cómo estas características son mediadas por los efectos individuales del estudiante y su entorno? En este marco, esta investigación tiene como propósito determinar perfiles de comportamiento de los elementos de la ecología del aprendizaje, en estudiantes de enseñanza superior, al participar en foro de discusión en un ambiente colaborativo de aprendizaje, considerando que el aprendizaje se puede producir en cualquier tiempo, espacio y/o lugar.

\section{OTROS ANTECEDENTES}

La educación superior está constantemente sufriendo transformaciones para integrar a sus estudiantes a la sociedad actual, que cada día está más globalizada y tecnologizada (Järvelä, 2019; Martínez, 2017; Avello y Duart, 2016). En este contexto surge la ecología del aprendizaje como una perspectiva integradora agregando un enfoque más social al aprendizaje, considerando que no hay lugar ni tiempo para aprender, ya que puede suceder en cualquier momento y/o lugar (Rocosa et al, 2018), es así como las investigaciones recientes muestran que entornos virtuales colaborativos de aprendizaje, cada vez son más utilizados (Hernández et al, 2019; Cornide-Reyes y Villarroel. 2019; Ludvigsen et al, 2017), principalmente debido a que, con el uso de la tecnología, se pueden potenciar las estrategias del aprendizaje colaborativo para facilitar procesos de aprendizaje efectivos en la educación superior, por sus características de interactividad, interacción, negociación y autorregulación, en cualquier tiempo y lugar (Martínez, 2018; Bodemer y Dehler, 
2011; Calvani et al, 2010), siendo el foro una de las herramientas más utilizadas en el aprendizaje colaborativo.

En el caso de los foros, es necesario destacar que los participantes utilicen de forma explícita y consensuada estrategias o habilidades de coordinación y colaboración. Este esfuerzo, favorece la adquisición de habilidades cognitivas y metacognitivas, las que juegan un papel esencial en los procesos de aprendizaje (Kupczynski et al, 2011; Dennen y Wieland, 2007). Se constata, por una parte, que a menudo los participantes no utilizan las posibilidades de comunicación y colaboración que ofrecen estas herramientas; y por otra, que la existencia de una participación elevada de intercambios comunicativos entre los participantes no conduce necesariamente al desarrollo de aprendizaje colaborativo, ni garantiza el logro de mejores resultados (Chávez et al, 2016). Desde el punto de vista metodológico, la diversidad y heterogeneidad de planteamientos no es menor. La investigación sobre el aprendizaje colaborativo, tanto en situaciones de interacción cara a cara como en entornos digitales, ha estado dividida entre lo cualitativo y lo cuantitativo (Strijbos et al, 2007). La situación, desde hace una década ha empezado a cambiar y cada vez son más frecuentes los trabajos que utilizan estrategias metodológicas multimétodo (Duke et al, 2018; Weinberger y Fisher, 2006).

Por otra parte, existe una corriente de investigación, que formula que hay que realizar una reconfiguración del aprendizaje hacia ecologías de aprendizaje (Martínez-Rodríguez et al, 2020; Coll, 2013). Las ecologías de aprendizaje comprenden variedad de procesos, contextos e interacciones que permiten al estudiante oportunidades y recursos para aprender, y así lograr alcanzar los logros declarados (González-Sanmamed et al, 2020). Lo que resulta consistente a la propuesta de entornos CSCL, y congruentes con los principios de la ecología del aprendizaje.

La metáfora ecológica se ha aplicado a muchos contextos y se adapta bien a las interacciones humanas entre las personas y su entorno, sus procesos para hacer, aprender y lograr, y para desarrollar nuevos conocimientos en contextos no estructurados (Martínez-Rodríguez et al, 2020). La ecología de aprendizaje de un individuo comprende su proceso y conjunto de contextos, relaciones e interacciones que brindan oportunidades y recursos para el aprendizaje, el desarrollo y los logros. Las ecologías de aprendizaje de creación propia son los medios por los cuales las experiencias y el aprendizaje se conectan e integran en los contextos y situaciones que constituyen la vida de una persona. Por lo tanto, las ecologías de aprendizaje tienen un valor conceptual y práctico significativo para la teoría y la práctica del aprendizaje y la educación a lo largo y ancho de la vida.

A lo largo, es lo tradicional que corresponde a los medios por los cuales conectamos e integramos nuestras experiencias y aprendizajes pasados y actuales, con una mirada hacia el futuro (Peters y Romero, 2019). Sin embargo, al considerar lo ancho, se abarcan múltiples dimensiones, como; los lugares y espacios físicos y virtuales que habitamos; y el significado que obtenemos de los contextos y situaciones que constituyen nuestras vidas. Las ecologías de aprendizaje son producto de la imaginación y la razón y son el vehículo de nuestros pensamientos y acciones creativas. Son uno de los sitios más importantes para la creatividad y nos permiten desarrollarnos personal y profesionalmente en todos los aspectos de nuestras vidas.

La educación tradicional no resiste este modelo, que requiere flexibilidad y múltiples interacciones, con la inserción de tecnología, se puede llegar a ambientes de aprendizaje de esta naturaleza, en particular la literatura apunta a los CSCL (Fatimah et al, 2021; Jara-Roa et al, 2019), que tiene varios de estos elementos incorporados al ser colaborativa y algunos de los no considerados se pueden intencionar, en este marco apunta la presente investigación, dónde los foros siguen siendo el foco del aprendizaje colaborativo y desde una mirada más amplia analizando el contexto dónde estos se desarrollan.

\section{METODOLOGÍA}

Para esta investigación se seleccionó una asignatura de primer año de enseñanza superior, así se garantizó la confiabilidad y validez de los datos, ya que la mayoría de los estudiantes de primer año no está contaminado con el sistema universitario. Se seleccionó la asignatura de Computación I, primer semestre 2018, cuyo resultado de aprendizaje fue modelar problemas simples e implementarlos en un lenguaje de alto nivel. La asignatura es semestral y al comienzo de ella, los y las estudiantes firman un consentimiento informado para; utilizar las contribuciones al foro; participación en grupos focales; y uso de sus notas finales, sin considerar sus nombres en las publicaciones. El curso constaba de 43 inscritos, donde el $15 \%$ son mujeres y la media de la edad es 20 años. Se utilizó un enfoque mixto del análisis de los datos que permita responder a las distintas preguntas de la investigación aprovechando las bondades y fortalezas de cada uno de ellos, para finalmente triangular su información.

La investigación, por estar inserta en una de orden mayor, cuenta con la aprobación del comité de ética institucional, quien tiene por objetivo principal examinar actividades de investigación institucionales, revisar informes y proyectos, en especial aquellos que tienen carácter de investigación científica en seres humanos, 
incluyendo ensayos clínicos, así como en animales, y en seres vivos, como también en aspectos tecnológicos y en todas las áreas de conocimientos que se desarrollan en esta Institución, desde la perspectiva ética, bioética y de bioseguridad.

El foro fue planificado considerando la filosofía de la ecología del aprendizaje y del trabajo colaborativo. Las características del foro fueron: a) carácter obligatorio, para garantizar contribuciones; el tema fue respecto a algoritmos simples, de forma general que le permitiera al estudiante variabilidad de contextos y contribuciones; b) la duración fue de tres semanas, para garantizar profundidad en contribuciones; c) intervalos mínimos de posteo, para garantizar contribuciones continuas en el tiempo y no todas al término del periodo; d) conversaciones sin límite de apertura, el/la estudiante decidía que conversación/tema abrir y en qué momento, la única condición es que una vez abierta la conversación/tema no se abriera otra equivalente, así se garantiza la personalización del foro hacia el estudiante; y, por último, e) hubo un incentivo en evaluación sumativa, para motivar participación.

La metodología que se utilizó para caracterizar los foros en ambientes colaborativo y de la ecología del aprendizaje en entornos virtuales, fue de carácter mixto (Johnson et al, 2007). El análisis cualitativo se realizó a partir de la propuesta de análisis de las contribuciones que realizan estudiantes de forma colaborativa y de grupos focales aplicados a estudiantes que participaron en el foro. Para clasificar las contribuciones de participantes en el foro se utiliza la rúbrica propuesta por Chávez et al (2016), donde se considera la naturaleza del contenido de acuerdo a una estructura jerárquica de cinco niveles ( $A$ : Análisis de información de pares; $P$ : Aporte de ideas; PC: Procesar el Contenido de Aprendizaje para llegar a una solución del problema; ST: Situar la tarea en un marco más amplio de conocimientos, experiencias e información; M: Motivación para cumplir la tarea) y su respectivo nivel de profundidad -escala Likert de 5 valoraciones-. Para indagar sobre la ecología del aprendizaje, se registró el día, la hora y los contextos de las conversaciones que abren los estudiantes en el foro.

El análisis cuantitativo consideró las categorizaciones obtenidas en la etapa anterior según el tipo de contribución que realizó el estudiante, el tamaño del mensaje, el día y hora que lo emite, además el rendimiento final que lograron los estudiantes del curso, realizando un análisis descriptivo e inferencial a través de árboles de clasificación que permitan determinar perfiles de actuaciones. Los árboles de clasificación (Breiman et al, 1984) es una técnica que permite clasificar, estratificar y/o pronosticar el tipo de mensaje que estamos propiciando en nuestros estudiantes. Esta labor de clasificar adquiere importancia si se dirige a crear directrices de planificación o en la elaboración de guías de actuación generales para crear procesos colaborativos. El proceso de clasificación se puede esquematizar en cuatro fases: construcción (building) del árbol, parada (stopping) del proceso de crecimiento del árbol, podado (pruning) del árbol haciéndolo más sencillo y dejando sólo los nodos más importantes y, por último, selección (selection) del árbol óptimo con capacidad de generalización.

Finalmente, para complementar los análisis, se triángulo la información realizando un grupo focal con los participantes del foro para indagar en mayor profundidad si la clasificación de los tipos de contribuciones de los mensajes, son consistentes a lo que opinan y perciben los estudiantes. Para ello, se seleccionaron 10 alumnos, distribuidos de la siguiente forma: 5 participantes activos; 2 no activos con contribuciones y 3 con sólo con contribuciones de baja jerarquía, correspondientes a las categorías A y P de la rúbrica.

\section{RESULTADOS}

La muestra estuvo formada por 43 estudiantes del curso Computación I, de los cuales, 26 participaron con contribuciones en el foro denominado: "Algoritmos Simples". Sin embargo, todos los estudiantes al menos vieron el foro cuatro veces. Las aportaciones totales fueron 192 y se abrieron 22 conversaciones, en un intervalo de tres semanas consecutivas. El foro se abre al segundo mes desde el inicio de clases. Las interacciones son altas, la conversación con menos post es de 4 y la mayor es con 18 aportaciones, siendo la media por conversación de 9 aportes.

Respecto a los resultados de la clasificación de las contribuciones al aplicar la rúbrica, se pueden observar en la figura 1, que la mayoría de las contribuciones son de tipo A, correspondiente a la categoría "análisis de información de pares", que está en la jerarquía más baja, aun así, las contribuciones se ubicaron en los niveles más profundos ( 3,4 y 5), la siguiente jerarquía baja es $P$ (aporte de ideas) dónde la mayoría de las contribuciones están en niveles de profundidad superficial (1, 2 y 3). La jerarquía más alta que se logra es ST, con contribuciones sólo en los niveles más altos de profundidad (3, 4 y 5). Otro de los aspectos, relacionados con las condiciones del tiempo, espacio y lugar, se muestra en la figura 2, la distribución respecto a la hora en la cual los estudiantes realizaban su contribución, según tipo de clasificación que logro dicho mensaje. 


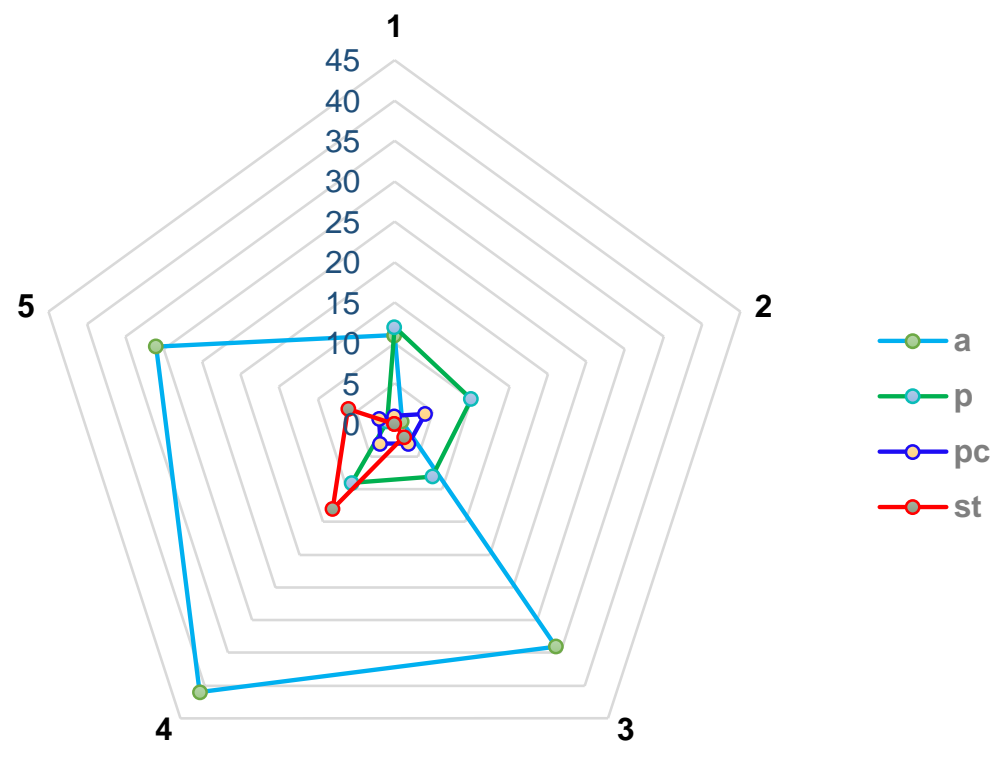

Fig. 1: Distribución de frecuencias de las contribuciones al foro por tipo de contribución y nivel de profundidad

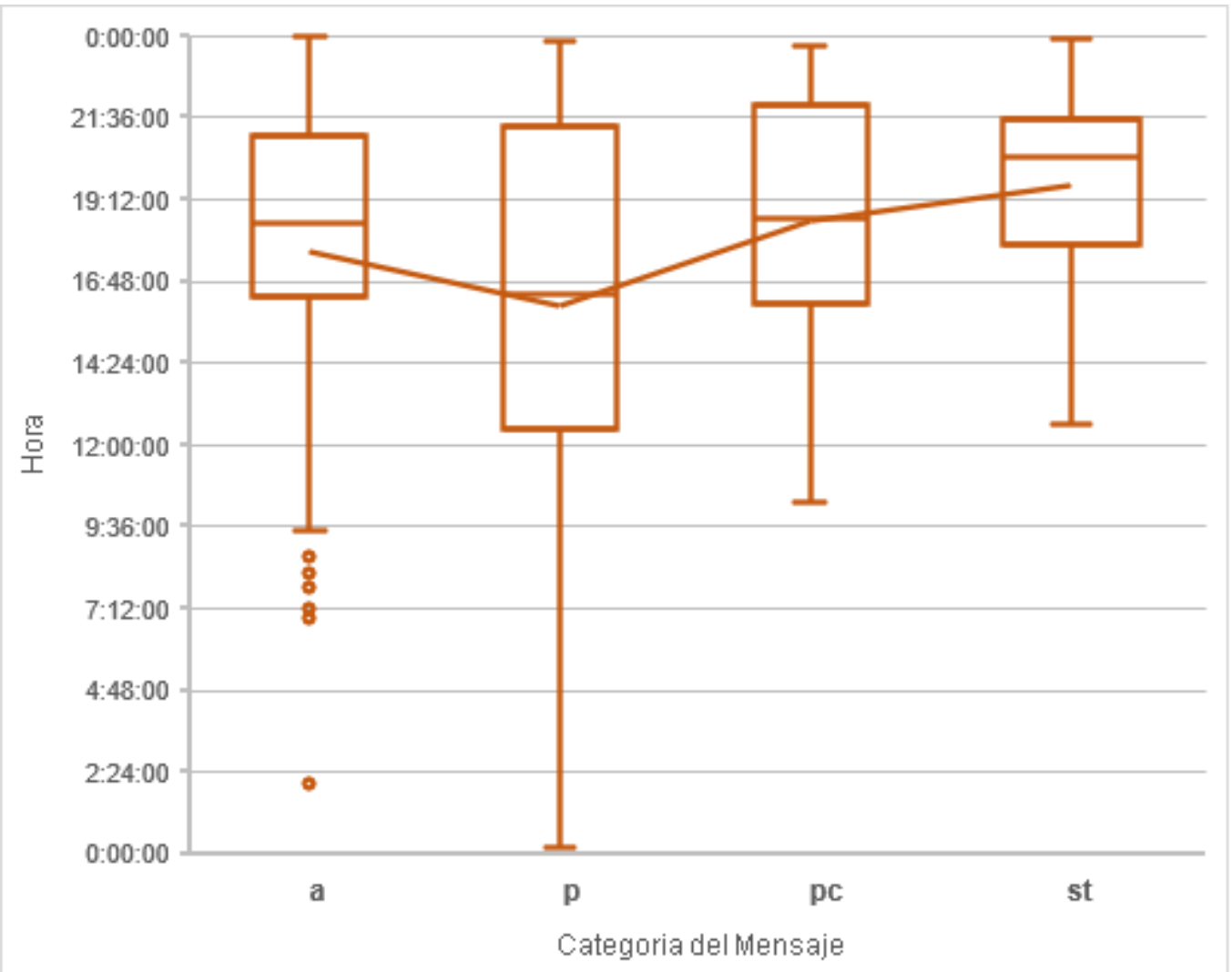

Fig. 2: Boxplot de la hora que los estudiantes emitieron los mensajes según su clasificación

La figura 2 representa los datos de los cuartiles, la media y horas atípicas en que los estudiantes realizaron las colaboraciones en el foro. En los mensajes tipo A y P (jerarquías más bajas) el horario de los aportes se realiza durante todo el día y el $50 \%$ de ellos hasta las 18:00 horas. En cambio, los mensajea tipo ST (alta jerarquía) se realizaron a partir de las 12:00 horas del día y el 50\% de ellos hasta las 20:30 horas. En esta misma perspectiva, otro elemento interesante corresponde a los días en los cuales escribe más el estudiante que realiza una contribución al foro. La figura 3 muestra que los días en que más se escribe son lunes, martes y jueves, y en los que menos se escribe son los viernes y sábado, en ese orden. Curiosamente el día domingo se escribe más que algunos días de semana, como el miércoles. 


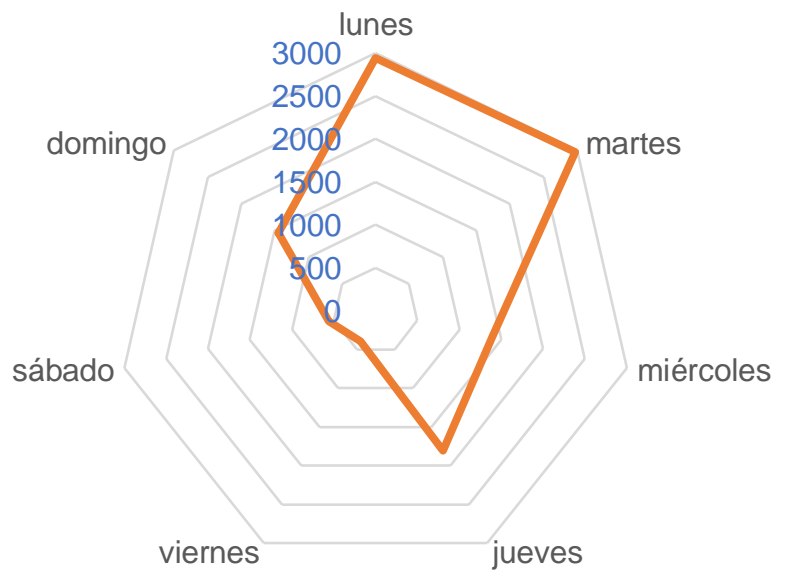

Fig. 3: Distribución de frecuencias de cantidad de palabras escritas por día.

Por otra parte, los aportes por conversaciones se pueden ver en la figura 4, estos se mueven en un intervalo de 4 a 18 aportes por conversación, el promedio de contribuciones por conversación es 9 , consistente con el tipo de interacción y de discusión de los temas que se abordaron. Una de las conversaciones que destaca es la numero 20 correspondiente al tema, "como usar una función en un algoritmo simple", que, por medio de ejemplos, los estudiantes intentan explicar a sus pares como se debe realizar una función con uso de parámetros y aquí se produce una dinámica con 18 post en total, donde los que intervienen mejoran un algoritmo, otros realizan preguntas para aclarar algo que no entienden, otros ocupan sentencias o librerías que permiten optimización y generalización, en suma, con esta conversación se puede ver que el tema tratado fue en profundidad, abarcando todos los aspectos relevantes a él.

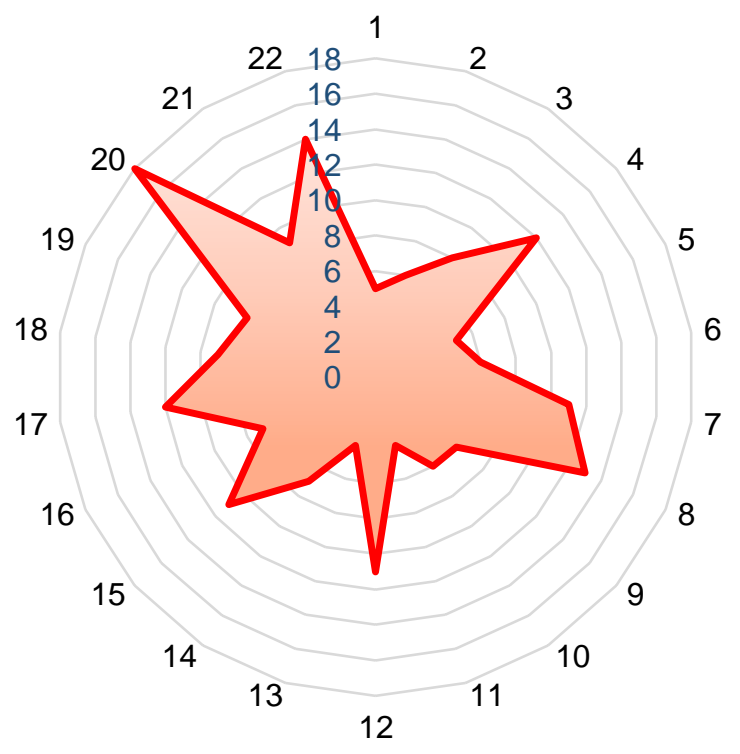

Fig. 4: Distribución de frecuencias de contribuciones por cada una de las 22 conversaciones

Para la caracterización final, el árbol óptimo de clasificación que muestra la figura 5, permitío generalizar algunas de las tendencias mostradas en los párrafos anteriores. Para entender el gráfico, la hora se trabaja en porcentaje, donde las 00:00 corresponde al $0 \%$ y las $23: 59$ al $100 \%$ respectivamente, por ejemplo medio día es $50 \%$ y las $18: 00 \mathrm{hrs}$. el $75 \%$. Algunas de las categorías de perfiles, que se extraen del árbol óptimo de clasificación, que son interesantes de destacar, corresponden a las siguientes: una contribución ST -alta jerarquía- se da cuando la contribución tiene más de 60 palabras, promedio de notas mayor a 5.1 y se escribe después de las 19:00 hrs.; una contribución de tipo A -baja jerarquía- tiene varias posibilidades, la más clara se da cuando la contribución tiene menos de 60 palabras y se escribe antes de las 16:30 hrs.; y, una contribución de tipo P -Baja jerarquía- se da cuando se escribe después de las 16:30 hrs., con una cantidad de palabras menor a 27 y los días martes, sábado y viernes. Además, es importante señalar que las notas tienen efecto sólo para categorizar las jerarquías de alto nivel, en este caso ST. 


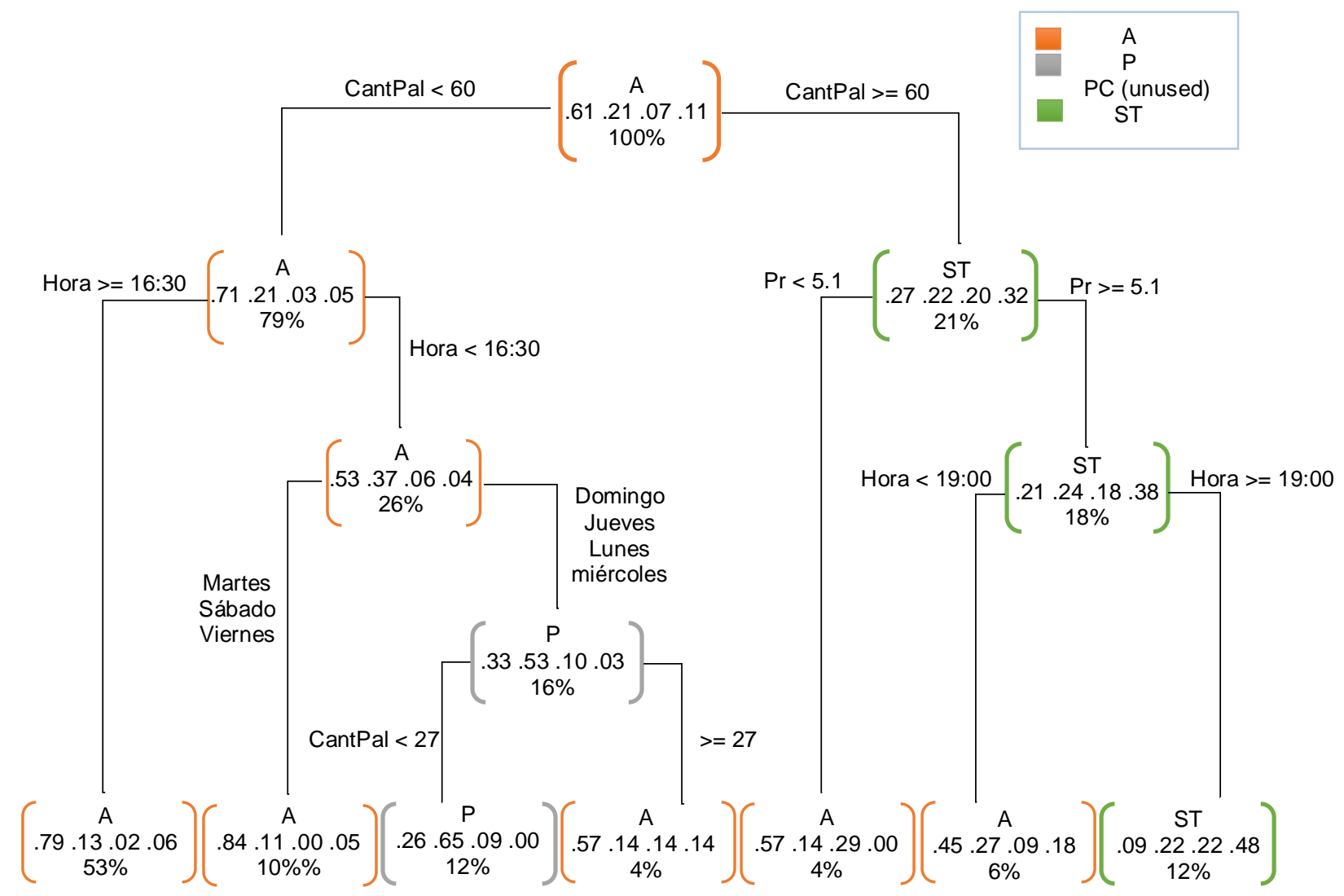

Fig. 5: Árbol de clasificación óptimo para las variables rendimiento, tipo de contribución, cantidad de palabras y hora en la que se produce la contribución.

Por último, se realizó un grupo focal, que nos sirvió para contrastar la información obtenida y tener algunos antecedentes que expliquen ciertos comportamientos, el grupo focal lo integraron 10 estudiantes, seleccionados de acuerdo al marco metodológico, algunos elementos emergentes importantes, entregados por los estudiantes, corresponden a: a) El alumno que no postea se frustra porque no encuentra que escribir, cuando va a realizar la contribución cree que la idea ya no se puede postear porque ya hay algo parecido en el foro y no sabe cómo redactarla de otra forma; b) Se hace muy largo a medida que avanza el foro, leer todos los comentarios para hacer una contribución significativa; c) El foro les sirvió más que en el momento, afirman haberlo ocupado después de su cierre para obtener ideas y soluciones a los problemas que se presentaban en laboratorio o trabajo; d) Es fácil hacer la primera réplica a una contribución, pero cuando hay cuatro o más, hay que "pensarla" bastante afirman; $y, d$ ) Uno de los aspectos que más les gusta, es que los ejemplos son libres, por eso pueden ejemplificar con elementos que ellos conocen y les gustan, como ejemplo hay algoritmos para el horóscopo chino, secuencias musicales, entre otras, es decir, elementos de la vida cotidiana, más cercanos y que les hace más sentido. En suma, los datos entregados en el foro son consistentes con la información global de la investigación, entregando algunos datos adicionales de contexto, que servirán para explicar algunos comportamientos del foro, por ejemplo, aquellos que no postearon o sus contribuciones fueron menores a las solicitadas.

\section{DISCUSIÓN}

El foro, en esta investigación, ratifica su relevancia cómo herramienta para el trabajo colaborativo y al mirarlo desde la perspectiva de la ecología del aprendizaje, nos entrega información relevante para entender cómo, cuándo y en qué lugar se produce el aprendizaje de los estudiantes y así fortalecer su proceso de enseñanza. Aun así, faltan elementos para dar una mirada global al aprendizaje personalizado. Uno de los aspectos más relevantes, es el tiempo en el cual, el estudiante produce una contribución de alto nivel, que no importa el día, pero que es después de las 19:00 hrs., si extrapolamos las investigaciones revisadas (González-Sanmamed et al, 2020; Hernández et al, 2019) en las cuales nos indican que el aprendizaje se debería producir cuando el alumno esté preparado, no en un horario fijo. Todo lo obtenido apunta a una educación más flexible con uso de tecnología, no necesariamente cien por ciento presencial, que respete los espacios de mayor creatividad, capacidad y todas las características de la ecología del aprendizaje focalizándose en la persona más que en el método. 
Aunque quedan estudios que realizar para generalizar, los resultados muestran que el viernes y sábado son los días que menos contribuciones se realizan en el foro, nos da un indicador del comportamiento social del estudiante, que es consistente con la vida cotidiana de los seres humanos en general. Nos muestra que el viernes lo deja para realizar actividades diferentes a las académicas. Esta conducta social, nos puede guiar para ponderar el tipo de actividad a realizar ciertos días y así ir potenciando el aprendizaje del estudiante.

Las percepciones entregadas por los estudiantes en el grupo focal son consistentes con los resultados globales de la investigación, en especial del árbol óptimo de clasificación, quien nos entregó información nueva y valiosa desde el punto de vista del estudiante, dónde se comienzan a apreciar atisbos de una intersección entre el aprendizaje y las características de la persona. Además, deja en evidencia, que la comunicación académica no es sencilla para el estudiante. Dado que quince de ellos no realizaron contribuciones, pero si miraron y leyeron el foro, pero no encontraron la forma de expresar sus ideas en el foro. Este es un aspecto a indagar en forma más profunda. La etapa de categorización de las contribuciones realizadas por los estudiantes es un proceso complejo en este tipo de investigaciones, su tratamiento es demasiado lento. Esto coincide con varias de las investigaciones revisadas (Martínez-Rodríguez et al, 2020; Coll, 2013) quienes abordan este problema y afirman que para dar un salto más en los ambientes CSCL y la ecología del aprendizaje hay que pensar en crear herramientas de clasificación automática, lo que con el avance que ha tenido la inteligencia artificial, no es una meta imposible de realizar.

Más específicamente, de acuerdo a los resultados obtenidos del estudio, se puede decir que: 1) se pueden obtener perfiles de los y las estudiantes de acuerdo a la profundidad del aporte, sus características y momento en el que se produce; 2) Los CSCL y la ecología del aprendizaje permiten al estudiante encontrar "el momento" que ellos elijan para participar, indudablemente, hay que indagar en más profundidad el motivo de su elección para potenciar aún más estos entornos de aprendizaje; y 3) Esta investigación, muestra que es relevante dar los espacios para que la o el estudiante pueda construir el conocimiento en el momento que decida, no impuesto por la rigidez del sistema actual, por lo que aportamos con información relevante, para remirar como se está realizando el proceso de enseñanza en educación superior, desde su perspectiva.

\section{CONCLUSIONES}

En términos generales, se concluye que la caracterización del foro, desde la perspectiva de los CSCL y la ecología del aprendizaje nos ha entregado información nueva que nos permite reorientar el proceso de enseñanza para potenciar las características personales de cada estudiante. Esto ha permitido establecer clasificaciones de perfil de comportamiento de los estudiantes, en las diferentes jerarquías de tipo de aprendizaje y profundidad, incidiendo en esta clasificación la hora, el día y la cantidad de palabras de la contribución.

\section{AGRADECIMIENTOS}

Los autores agradecen el apoyo del Proyecto Dicyt, código 041733BC, Vicerrectoría de Investigación. Desarrollo e Innovación de la Universidad de Santiago de Chile.

\section{REFERENCIAS}

Arras-Vota, A.M., Bordas-Beltrán, J.L., Porras-Flores, D.A., y Gómez-Ramírez, J.I., Competencias en tecnologías de información y comunicación. Estudios de caso: Universidad Santo Tomas (Colombia) y Universidad Autónoma de Chihuahua (México), http://dx.doi.org/10.4067/S0718-50062021000100135, Formación Universitaria, 14, 1 (2021)

Avello R., y Duart, J., Nuevas tendencias de aprendizaje colaborativo en e-learning. Claves para su implementación efectiva, http://dx.doi.org/10.4067/S0718-07052016000100017, Estudios Pedagógicos, 42, 1 (2016)

Bodemer, D., y Dehler, J., Group awareness in CSCL environments, https://doi.org/10.1016/j.chb.2010.07.014, Computers in Human Behavior, 27(3), 1043-1045 (2011)

Breiman, L., Friedman, J.H., Olshen, R.A., y Stone, C.J., Classification and regression trees, $1^{\text {a }}$ edition, Editorial Taylor \& Francis Group (1984)

Calvani, A., Fini, A., Molino, M., y Ranieri, M., Visualizing and monitoring effective interactions in online collaborative groups, doi:10.1111/j.1467-8535.2008.00911.x, British Journal of Educational Technology, 41(2), $213-226$ (2010)

Chávez, J., Montaño, R., y Barrera, R., Structure and content of messages in an online environment: an approach from participation, https://doi.org/10.1016/j.chb.2015.08.046, Computers in Human Behavior, 54(1), 560-568 (2016)

Coll, C., El currículo escolar en el marco de la nueva ecología del aprendizaje, reflexión, Revista Aula, 219, 31-36 (2013)

Cornide-Reyes, H., y Villarroel, R., Método para promover el aprendizaje colaborativo en ingeniería de software, http://dx.doi.org/10.4067/S0718-50062019000400003, Formación Universitaria, 12(4), 3-12 (2019) 
Dado, M., y Bodemer, D., A review of methodological applications of social network analysis in computer-supported collaborative learning, https://doi.org/10.1016/j.edurev.2017.08.005, Educational Research Review, 22, 159-180 (2017)

Dennen, V.P., y Wieland, K., From interaction to intersubjectivity: facilitating online group discourse processes, https://doi.org/10.1080/01587910701611328, Distance Education, 28(3), 281-297 (2007)

Duque, R., Isotani, S., y otros cuatro autores, Affective states in computer-supported collaborative learning: studying the past to drive the future, https://doi.org/10.1016/j.compedu.2018.01.015, Computers \& Education, 120, 29-50 (2018)

Fatimah, F., Rajiani, S., y Abbas, E., Cultural and individual characteristics in adopting computer-supported collaborative learning during covid-19 outbreak: Willingness or obligatory to accept technology?, doi:10.5267/j.msl.2020.9.032, Management Science Letters, 11(2), 373-378 (2021)

González-Sanmamed, M., Sangrà, A., Souto-Seijo, A., y Estévez, I., Learning ecologies in the digital era: challenges for higher education, doi:10.30827/publicaciones.v50i1.15671, Publicaciones, 50(1), 83-102 (2020)

Hernández, N., Muñoz-Carril, P., y González-Sanmamed, M., Computer-supported collaborative learning: an analysis of the relationship between interaction emotional support and online collaborative tools, https://doi.org/10.1016/j.compedu.2019.04.012, Computers \& Education, 138, 1-12 (2019)

Jara-Roa, D., Ramírez-Montoya, M., Cabezas, M., y Real-Deus, J., Predicción del desempeño en una tarea colaborativa con uso de tecnologías. El papel de la construcción social del conocimiento y la comunicación, Revista lbérica de Sistemas e Tecnologias de Informação, E22, 134-146 (2019)

Järvelä, S., Järvenoja, H., y Malmberg, J., Capturing the dynamic and cyclical nature of regulation: methodological progress in understanding socially shared regulation in learning, doi: 10.1007/s11412-019-09313-2, ijcscl, 14 (4), 425$441(2019)$

Johnson, R. B., Onwuegbuzie, A. J., y Turner, L. A., Toward a definition of mixed methods research, doi:10.1177/1558689806298224, Journal of Mixed Methods Research, 1(2), 112-133 (2007)

Kupczynski, L., Gibson, A. M., y otros tres autores, The impact of frequency on achievement in online courses: a study from a South Texas University, Journal of Interactive Online Learning, 10(3), 141-149 (2011)

Ludvigsen, S., Cress, U., y otros tres autores, Future direction for the CSCL field: methodologies and eight controversies, doi: 10.1007/s11412-017-9268-4, ijcscl, 12 (4), 337-34 (2017)

Martínez, G.A., Diseño de una guía didáctica basada en la integración de mundos virtuales al entorno educativo de la Universidad de Cundinamarca, http://dx.doi.org/10.4067/S0718-50062017000100002, Formación Universitaria, 10(1) (2017)

Martínez, O., Steffens, E.J., Ojeda D.C., y Hernández, H.G., Estrategias pedagógicas aplicadas a la educación con mediación virtual para la generación del conocimiento global, http://dx.doi.org/10.4067/S0718-50062018000500011, Formación Universitaria, 11, 5 (2018)

Martínez-Rodríguez, R., y Benítez-Corona, L., The ecology of resilience learning in ubiquitous environments to adverse situations, doi:10.3916/C62-2020-04, Comunicar English ed., 28(62), 43-52 (2020)

Peters, M., y Romero, M., Lifelong learning ecologies in online higher education: students' engagement in the continuum between formal and informal learning, https://doi-org.ezproxy.usach.cl/10.1111/bjet.12803, British Journal of Educational Technology, 50(4), 1729-1743 (2019)

Radkowitsch, A., Vogel, F., y Fischer, F., Good for learning, bad for motivation? a meta-analysis on the effects of computer-supported collaboration scripts, doi: 10.1007/s11412-020-09316-4, ijcscl 15 (1), 5-47 (2020)

Rocosa, B., Sangrà, A., y Cabrera, N., La organización escolar y el desarrollo de la competencia de aprender a aprender: un enfoque globalizador singular, doi: 10.21703/rexe.Especial2_201831512, REXE, Revista de Estudios y Experiencias en Educación, 2, 31-51 (2018)

Stahl, G., A decade of CSCL international, doi: 10.1007/s11412-015-9222-2, Journal of Computer-Supported Collaborative Learning, 10(4), 337-344 (2015)

Strijbos, J. W., Martens, R. L., Jochems, W. M. G., y Broers, N. J., The effect of functional roles on perceived group efficiency during computer-supported collaborative learning: a matter of triangulation, https://doi.org/10.1016/j.chb.2004.10.016, Computers in Human Behavior, 23, 353-380 (2007)

VanOostveen, R., Desjardins, F., y Bullock, S., Professional development learning environments (PDLEs) embedded in a collaborative online learning environment (COLE): moving towards a new conception of online professional learning, doi:10.1007/s10639-018-9686-6, Education and Information Technologies, 24(2), 1863-1900 (2019)

Weinberger, A., y Fischer, F., A framework to analyze argumentative knowledge construction in computer-supported collaborative learning, https://doi.org/10.1016/j.compedu.2005.04.003, Computers \& Education, 46, 71-95 (2006) 
